\title{
Monotone iterative procedure and systems of a finite number of nonlinear fractional differential equations
}

\section{Dariusz Wardowski}

\section{"Correspondence:}

wardd@math.uni.lodz.pl Department of Nonlinear Analysis, Faculty of Mathematics and Computer Science, University of Łódź, Banacha 22, Łódź, 90-238, Poland

\begin{abstract}
The aim of the paper is to present a nontrivial and natural extension of the comparison result and the monotone iterative procedure based on upper and lower solutions, which were recently established in (Wang et al. in Appl. Math. Lett. 25:1019-1024, 2012), to the case of any finite number of nonlinear fractional differential equations.
\end{abstract}

MSC: 26A33; 34A08; 34B15

Keywords: monotone iterative procedure; system of fractional differential equations; upper and lower solution

\section{Introduction}

Fractional derivatives and integrals are used for a better description of material properties. In the literature we can find many interesting papers concerning this theory; see e.g., [1-13]. The study of systems involving fractional differential/integral equations is also important as such systems occur in various problems of applied nature; for example, see [1422]. Some basic theory of fractional differential equations involving the Riemann-Liouville differential operator can be found in [23-25].

In the paper we consider the following system of nonlinear fractional differential equations:

$$
\left\{\begin{array}{l}
D^{\alpha} u_{1}(t)=f_{1}\left(t, u_{1}(t), u_{2}(t), \ldots, u_{n}(t)\right), \quad t \in(0, T], \\
D^{\alpha} u_{2}(t)=f_{2}\left(t, u_{1}(t), u_{2}(t), \ldots, u_{n}(t)\right), \quad t \in(0, T], \\
\ldots, \\
D^{\alpha} u_{n}(t)=f_{n}\left(t, u_{1}(t), u_{2}(t), \ldots, u_{n}(t)\right), \quad t \in(0, T], \\
\left.t^{1-\alpha} u_{1}(t)\right|_{t=0}=x_{0}^{1},\left.\quad t^{1-\alpha} u_{2}(t)\right|_{t=0}=x_{0}^{2}, \quad \ldots,\left.\quad t^{1-\alpha} u_{n}(t)\right|_{t=0}=x_{0}^{n},
\end{array}\right.
$$

where $D^{\alpha}$ is the standard Riemann-Liouville fractional derivative of order $\alpha, 0 \leq \alpha \leq 1$, $T>0, f^{i} \in C\left([0, T] \times \mathbb{R}^{n}, \mathbb{R}\right), 1 \leq i \leq n$, and $x_{0}^{1}, \ldots, x_{0}^{n} \in \mathbb{R}$ satisfy

$$
\sum_{i=2}^{n} x_{0}^{i}-x_{0}^{1} \geq 0
$$

\section{Springer}


We investigate system (1.1) with respect to the existence of a solution via the method of upper and lower solutions. There is also presented the concept of an iterative procedure, where the appropriately constructed sequences are convergent to the extreme solution. The paper is a continuation of the investigations in [10] of Wang et al., where the authors examined system (1.1) in the case $n=2$. After proving the main results we state, for convenience of the reader, the introduced techniques in the case of three nonlinear fractional differential equations and also present a concrete example.

\section{Preliminaries}

First, let us recall the needed notations and crucial results which will be needed in the next sections of the article.

Denote by $C_{1-\alpha}([0, T])$ the family of all functions $u \in C((0, T])$ such that $t^{1-\alpha} u \in$ $C([0, T])$. A basic theorem concerning the existence of the result and its uniqueness for the linear fractional equation is as follows.

Lemma 2.1 ([23]) Let $0<\alpha \leq 1, M \in \mathbb{R}$, and $\sigma \in C_{1-\alpha}([0, T])$ be fixed. Then the linear initial value problem

$$
\left\{\begin{array}{l}
D^{\alpha} u(t)=\sigma(t)-M u(t), \quad t \in(0, T], \quad i, j \in \mathbb{N}, \\
\left.t^{1-\alpha} u(t)\right|_{t=0}=u_{0},
\end{array}\right.
$$

has a unique solution, given by the following formula:

$$
u(t)=\Gamma(\alpha) u_{0} t^{\alpha-1} E_{\alpha, \alpha}\left(-M t^{\alpha}\right)+\int_{0}^{t}(t-s)^{\alpha-1} E_{\alpha, \alpha}\left(-M(t-s)^{\alpha}\right) \sigma(s) d s,
$$

where $E_{\alpha, \beta}$ is the Mittag-Leffler function, i.e. the function of the form

$$
E_{\alpha, \beta}(z)=\sum_{k=0}^{\infty} \frac{z^{k}}{\Gamma(\alpha k+\beta)}, \quad \alpha, \beta>0, z \in \mathbb{R} .
$$

The comparison result for the initial value problem (2.1) due to Wang et al. is as follows.

Lemma 2.2 ([10]) Let $0<\alpha \leq 1$ and $M \in \mathbb{R}$ be given. Then, if $w \in C_{1-\alpha}([0, T])$ satisfies

$$
\left\{\begin{array}{l}
D^{\alpha} w(t)+M w(t) \geq 0, \quad t \in(0, T] \\
\left.t^{1-\alpha} w(t)\right|_{t=0} \geq 0
\end{array}\right.
$$

then $w(t) \geq 0$ for all $t \in(0, T]$.

The same authors also proved the following result, which will be needed in the sequel.

Lemma 2.3 ([10]) Let $0<\alpha \leq 1, M \in \mathbb{R}$, and $N \geq 0$ be given. Assume that $u, v \in$ $C_{1-\alpha}([0, T])$ satisfy

$$
\left\{\begin{array}{l}
D^{\alpha} u(t) \geq-M u(t)+N v(t), \quad t \in(0, T], \\
D^{\alpha} v(t) \geq-M v(t)+N u(t), \quad t \in(0, T], \\
\left.t^{1-\alpha} u(t)\right|_{t=0} \geq 0,\left.\quad t^{1-\alpha} v(t)\right|_{t=0} \geq 0 .
\end{array}\right.
$$

Then $u(t) \geq 0, v(t) \geq 0$ for all $t \in(0, T]$. 


\section{The results}

In the sequel we will use the following notation:

$$
\delta_{i j}=\left\{\begin{array}{ll}
1 & \text { if } i=j, \\
-1 & \text { if } i \neq j,
\end{array} \quad i, j \in \mathbb{N} .\right.
$$

$C_{1-\alpha}([0, T])^{n}$ denotes $C_{1-\alpha}([0, T]) \times C_{1-\alpha}([0, T]) \times \cdots \times C_{1-\alpha}([0, T])(n$ times $)$.

Lemma 3.1 Let $0<\alpha \leq 1$ be fixed, $M_{i} \in \mathbb{R}, \sigma_{i} \in C_{1-\alpha}([0, T]), i=1,2, \ldots, n$. Then the linear problem of $n$ equations

$$
\left\{\begin{aligned}
& D^{\alpha} u_{1}(t)=\sigma_{1}(t)-M_{1} u_{1}(t)-\sum_{i, j=2}^{n} M_{j} \delta_{j i} u_{i}(t), \quad t \in(0, T] \\
& D^{\alpha} u_{j}(t)= \sigma_{j}(t)+\left(M_{j}-\sum_{i=1}^{n} M_{i}\right) u_{j}(t) \\
&-M_{j}\left(\sum_{i=1}^{n} u_{i}(t)-u_{j}(t)\right), \quad t \in(0, T], 2 \leq j \leq n \\
&\left.t^{1-\alpha} u_{i}(t)\right|_{t=0}=x_{0}^{i}, \quad 1 \leq i \leq n
\end{aligned}\right.
$$

has a unique solution in $C_{1-\alpha}([0, T])^{n}$.

Proof First observe that for any $p_{1}, p_{2}, \ldots, p_{n} \in C_{1-\alpha}([0, T])$ the system

$$
\left\{\begin{array}{l}
u_{1}+u_{2}+\cdots+u_{n}=p_{1} \\
u_{1}-u_{2}+\cdots+u_{n}=p_{2} \\
\cdots \\
u_{1}+u_{2}+\cdots-u_{n}=p_{n}
\end{array}\right.
$$

has exactly one solution, which is a consequence of the fact that

$$
\operatorname{det}\left[\begin{array}{ccccc}
1 & 1 & 1 & \cdots & 1 \\
1 & -1 & 1 & \cdots & 1 \\
1 & 1 & -1 & \cdots & 1 \\
\vdots & \vdots & \vdots & \ddots & \vdots \\
1 & 1 & 1 & \cdots & -1
\end{array}\right]_{n \times n}=(-2)^{n-1} \neq 0
$$

Next, observe that system (3.1) can be transformed to system (3.2), where $p_{1}, p_{2}, \ldots, p_{n}$ solve the following $n$ problems:

$$
\begin{aligned}
& \left\{\begin{array}{l}
D^{\alpha} p_{1}(t)=\left(\sigma_{1}(t)+\sigma_{2}(t)+\cdots+\sigma_{n}(t)\right)-\left(M_{1}+M_{2}+\cdots+M_{n}\right) p_{1}(t), \\
\left.t^{1-\alpha} p_{1}(t)\right|_{t=0}=x_{0}^{1}+x_{0}^{2}+\cdots+x_{0}^{n},
\end{array}\right. \\
& \left\{\begin{array}{l}
D^{\alpha} p_{2}(t)=\left(\sigma_{1}(t)-\sigma_{2}(t)+\cdots+\sigma_{n}(t)\right)-\left(M_{1}-M_{2}+\cdots+M_{n}\right) p_{2}(t), \\
\left.t^{1-\alpha} p_{2}(t)\right|_{t=0}=x_{0}^{1}-x_{0}^{2}+\cdots+x_{0}^{n},
\end{array}\right. \\
& \vdots \\
& \left\{\begin{array}{l}
D^{\alpha} p_{n}(t)=\left(\sigma_{1}(t)+\sigma_{2}(t)+\cdots-\sigma_{n}(t)\right)-\left(M_{1}+M_{2}+\cdots-M_{n}\right) p_{n}(t), \\
\left.t^{1-\alpha} p_{n}(t)\right|_{t=0}=x_{0}^{1}+x_{0}^{2}+\cdots-x_{0}^{n} .
\end{array}\right.
\end{aligned}
$$

Finally, observe that the solutions of the above equations are unique due to Lemma 2.1, which ends the proof. 
Now we can state and proof the comparison result for system (3.1).

Theorem 3.1 Let $0<\alpha \leq 1, M_{1} \in \mathbb{R}, M_{2}, \ldots, M_{n} \geq 0$, and let $u_{1}, \ldots, u_{n} \in C_{1-\alpha}([0, T])$ satisfy

$$
\left\{\begin{aligned}
& D^{\alpha} u_{1}(t) \geq-M_{1} u_{1}(t)+\sum_{i, j=2}^{n} M_{j} \delta_{j i} u_{i}(t), \quad t \in(0, T] \\
& D^{\alpha} u_{s}(t) \geq-M_{1} u_{s}(t)+\left(\sum_{i=2}^{n} M_{i}-M_{s}\right) u_{s}(t) \\
&+M_{s}\left(\sum_{i=1}^{n} u_{i}(t)-u_{s}(t)\right), \quad 2 \leq s \leq n, t \in(0, T] \\
&\left.t^{1-\alpha} u_{s}(t)\right|_{t=0} \geq 0, \quad 1 \leq s \leq n
\end{aligned}\right.
$$

Then

$$
\begin{aligned}
& \sum_{i=1}^{n} u_{i}(t) \geq 0, \quad t \in(0, T] \\
& u_{s}(t) \geq 0, \quad t \in(0, T], 2 \leq s \leq n, \\
& -u_{s}(t)+\sum_{i=1}^{n} u_{i}(t) \geq 0, \quad t \in(0, T], 2 \leq s \leq n .
\end{aligned}
$$

Proof Put $r(t)=\sum_{s=1}^{n} u_{s}(t)$. Using (3.3) we obtain

$$
\begin{aligned}
D^{\alpha} r(t)= & \sum_{s=1}^{n} D^{\alpha} u_{s}(t) \\
\geq & -M_{1} u_{1}(t)+\sum_{i, j=2}^{n} M_{j} \delta_{j i} u_{i}(t)-M_{1} \sum_{s=2}^{n} u_{s}(t)-2 \sum_{s=2}^{n} M_{s} u_{s}(t) \\
& +\sum_{s=2}^{n} \sum_{i=2}^{n} M_{i} u_{s}(t)+\sum_{s=2}^{n} \sum_{i=1}^{n} M_{s} u_{i}(t) \\
= & -M_{1} r(t)+\sum_{i, j=2}^{n}\left(M_{j} \delta_{j i} u_{i}(t)+M_{i} u_{j}(t)\right)-2 \sum_{s=2}^{n} M_{s} u_{s}(t)+\sum_{s=2}^{n} M_{s} r(t) .
\end{aligned}
$$

Observe that

$$
\sum_{i, j=2, i \neq j}^{n}\left(M_{j} \delta_{j i} u_{i}(t)+M_{i} u_{j}(t)\right)=0 .
$$

Hence, we obtain

$$
\begin{aligned}
D^{\alpha} r(t) \geq & -\left(M_{1}-\sum_{s=2}^{n} M_{s}\right) r(t)+\sum_{i, j=2, i \neq j}^{n}\left(M_{j} \delta_{j i} u_{i}(t)+M_{i} u_{j}(t)\right) \\
& +\sum_{i, j=2, i=j}^{n}\left(M_{j} \delta_{j i} u_{i}(t)+M_{i} u_{j}(t)\right)-2 \sum_{s=2}^{n} M_{s} u_{s}(t) \\
= & -\left(M_{1}-\sum_{s=2}^{n} M_{s}\right) r(t)+2 \sum_{i=2}^{n} M_{i} u_{i}(t)-2 \sum_{s=2}^{n} M_{s} u_{s}(t) \\
= & -\left(M_{1}-\sum_{s=2}^{n} M_{s}\right) r(t) .
\end{aligned}
$$


Moreover, observe that

$$
t^{1-\alpha} r(t)=\sum_{s=1}^{n} t^{1-\alpha} u_{s}(t) \geq 0
$$

Applying (3.8) and (3.9) to Lemma 2.2 we get (3.4).

Now, consider any $2 \leq s \leq n$ and denote

$$
r_{s}(t)=\sum_{i=1}^{n} u_{i}(t)-u_{s}(t), \quad t \in(0, T]
$$

By (3.3) we have

$$
\begin{aligned}
D^{\alpha} r_{s}(t)= & \sum_{i=1}^{n} D^{\alpha} u_{i}(t)-D^{\alpha} u_{s}(t)=D^{\alpha} u_{1}(t)+\sum_{i=2}^{n} D^{\alpha} u_{i}(t)-D^{\alpha} u_{s}(t) \\
\geq & -M_{1} u_{1}(t)+\sum_{i, j=2}^{n} M_{j} \delta_{j i} u_{i}(t)-\sum_{i=2}^{n} M_{1} u_{i}(t)+M_{1} u_{s}(t) \\
& +\sum_{i=2}^{n}\left(\sum_{j=2}^{n} M_{j}-M_{i}\right) u_{i}(t)-\left(\sum_{j=2}^{n} M_{j}-M_{s}\right) u_{s}(t) \\
& +\sum_{i=2}^{n} M_{i}\left(\sum_{j=1}^{n} u_{j}(t)-u_{i}(t)\right)-M_{s}\left(\sum_{j=1}^{n} u_{j}(t)-u_{s}(t)\right) \\
= & -M_{1} u_{1}(t)+\sum_{i, j=2}^{n}\left(M_{j} \delta_{j i} u_{i}(t)+M_{j} u_{i}(t)\right)-M_{1} \sum_{i=2}^{n} u_{i}(t)+M_{1} u_{s}(t)+M_{s} u_{s}(t) \\
& -2 \sum_{i=2}^{n} M_{i} u_{i}(t)-u_{s}(t) \sum_{j=2}^{n} M_{j}+\sum_{i=2}^{n} \sum_{j=1}^{n} M_{i} u_{j}(t)-M_{s} r_{s}(t) .
\end{aligned}
$$

Again, using (3.7), we obtain

$$
\begin{aligned}
D^{\alpha} r_{s}(t) \geq & -M_{1} \sum_{i=1}^{n} u_{i}(t)+M_{1} u_{s}(t)+M_{s} u_{s}(t)-u_{s}(t) \sum_{j=2}^{n} M_{j} \\
& +\sum_{i=2}^{n} M_{i} r_{s}(t)+u_{s}(t) \sum_{i=2}^{n} M_{i}-M_{s} r_{s}(t) \\
= & -\left(M_{1}-\sum_{i=2}^{n} M_{i}+M_{s}\right) r_{s}(t)+M_{s} u_{s}(t) .
\end{aligned}
$$

Moreover, observe that (3.3) implies

$$
D^{\alpha} u_{s}(t) \geq-\left(M_{1}-\sum_{i=2}^{n} M_{i}+M_{s}\right) u_{s}(t)+M_{s} r_{s}(t) .
$$

Finally, note that (3.10) and (3.11) applied to Lemma 2.3 give (3.5) and (3.6).

Now, we are in a position to enunciate the main result. 
Theorem 3.2 Suppose that there exist $u_{0}^{1}, u_{0}^{2}, \ldots, u_{0}^{n} \in C_{1-\alpha}([0, T]), u_{0}^{1} \leq \sum_{i=2}^{n} u_{0}^{i}$, satisfying

$$
\begin{cases}D^{\alpha} u_{0}^{1}(t) \leq f_{1}\left(t, u_{0}^{1}(t), u_{0}^{2}(t), \ldots, u_{0}^{n}(t)\right), & t \in(0, T], \\ D^{\alpha} u_{0}^{s}(t) \geq f_{s}\left(t, u_{0}^{1}(t), u_{0}^{2}(t), \ldots, u_{0}^{n}(t)\right), & t \in(0, T], 2 \leq s \leq n, \\ \left.t^{1-\alpha} u_{0}^{1}(t)\right|_{t=0} \leq x_{0}^{1}, & \\ \left.t^{1-\alpha} u_{0}^{s}(t)\right|_{t=0} \geq x_{0}^{s}, \quad 2 \leq s \leq n, & \end{cases}
$$

and there exist $M_{1} \in \mathbb{R}, M_{2}, \ldots, M_{n}>0$ such that

(i)

$$
f_{1}\left(t, \alpha_{1}, \ldots, \alpha_{n}\right)-f_{1}\left(t, \beta_{1}, \ldots, \beta_{n}\right) \geq-M_{1}\left(\alpha_{1}-\beta_{1}\right)-\sum_{i, j=2}^{n} M_{j} \delta_{j i}\left(\alpha_{i}-\beta_{i}\right)
$$

(ii)

$$
\begin{aligned}
& f_{s}\left(t, \alpha_{1}, \ldots, \alpha_{n}\right)-f_{s}\left(t, \beta_{1}, \ldots, \beta_{n}\right) \\
& \quad \geq\left(-M_{1}+\sum_{i=2}^{n} M_{i}-M_{s}\right)\left(\alpha_{s}-\beta_{s}\right)-M_{s}\left(\alpha_{1}-\beta_{1}+\alpha_{s}-\beta_{s}-\sum_{i=2}^{n}\left(\alpha_{i}-\beta_{i}\right)\right),
\end{aligned}
$$

where $\alpha_{i}, \beta_{i} \in \mathbb{R}, 1 \leq i \leq n$ satisfy for all $t \in[0, T]$ and $2 \leq s \leq n$,

$$
\begin{aligned}
& u_{0}^{1}(t)-\left(\sum_{i=2}^{n} u_{0}^{i}(t)-u_{0}^{s}(t)\right) \leq \beta_{1}-\left(\sum_{i=2}^{n} \beta_{i}-\beta_{s}\right) \leq \alpha_{1}-\left(\sum_{i=2}^{n} \alpha_{i}-\alpha_{s}\right) \leq u_{0}^{s}(t) \\
& u_{0}^{1}(t)-\left(\sum_{i=2}^{n} u_{0}^{i}(t)-u_{0}^{s}(t)\right) \leq \alpha_{s} \leq \beta_{s} \leq u_{0}^{s}(t)
\end{aligned}
$$

(iii)

$$
\begin{aligned}
& \sum_{s=2}^{n} f_{s}\left(t, u^{1}(t), u^{2}(t), \ldots, u^{n}(t)\right)-f_{1}\left(t, u^{1}(t), u^{2}(t), \ldots, u^{n}(t)\right) \\
& \quad \geq\left(-M_{1}+\sum_{s=2}^{n} M_{s}\right)\left(\sum_{s=2}^{n} u^{s}(t)-u^{1}(t)\right)
\end{aligned}
$$

where

$$
u_{0}^{1}-\left(\sum_{i=2}^{n} u_{0}^{i}-u_{0}^{s}\right) \leq u^{1}-\left(\sum_{i=2}^{n} u^{i}-u^{s}\right) \leq u^{s} \leq u_{0}^{s}, \quad 2 \leq s \leq n
$$

Then there exists a solution $\left(\bar{u}^{1}, \bar{u}^{2}, \ldots, \bar{u}^{n}\right)$ of system (1.1) such that

$$
(n-1) u_{0}^{1}-(n-2) \sum_{i=2}^{n} u_{0}^{i} \leq \bar{u}^{1} \leq \sum_{i=2}^{n} u_{0}^{i}, \quad u_{0}^{1}-\sum_{i=2}^{n} u_{0}^{i}+u_{0}^{s} \leq \bar{u}^{s} \leq u_{0}^{s}, \quad 2 \leq s \leq n
$$

Moreover, there exist iterative sequences $\left(u_{k}^{1}\right),\left(u_{k}^{2}\right), \ldots,\left(u_{k}^{n}\right)$ such that $u_{k}^{i} \rightarrow \bar{u}^{i}, k \rightarrow \infty, i=$ $1,2, \ldots, n$, uniformly on compact subsets of $(0, T]$. 
Proof Let us first consider the linear system of the form

$$
\left\{\begin{aligned}
& D^{\alpha} u^{1}(t)= f_{1}\left(t, u_{0}^{1}(t), u_{0}^{2}(t), \ldots, u_{0}^{n}(t)\right)+M_{1} u_{0}^{1}(t)+\sum_{i, j=2}^{n} M_{j} \delta_{j i} u_{0}^{i}(t) \\
&-M_{1} u^{1}(t)-\sum_{i, j=2}^{n} M_{j} \delta_{j i} u^{i}(t), \quad t \in(0, T] \\
& D^{\alpha} u^{s}(t)= f_{s}\left(t, u_{0}^{1}(t), u_{0}^{2}(t), \ldots, u_{0}^{n}(t)\right)+M_{1} u_{0}^{s}(t)+\left(\sum_{i=2}^{n} M_{i}-M_{s}\right) u^{s}(t) \\
&+M_{s}\left(u_{0}^{1}(t)+\sum_{i=2}^{n} u^{i}(t)-u^{s}(t)\right)-M_{1} u^{s}(t)-\left(\sum_{i=2}^{n} M_{i}-M_{s}\right) u_{0}^{s}(t) \\
&-M_{s}\left(u^{1}(t)+\sum_{i=2}^{n} u_{0}^{i}(t)-u_{0}^{s}(t)\right), \quad t \in(0, T], 2 \leq s \leq n, \\
&\left.t^{1-\alpha} u^{s}(t)\right|_{t=0}=x_{0}^{s}, \quad 1 \leq s \leq n,
\end{aligned}\right.
$$

where $u^{1}, u^{2}, \ldots, u^{n} \in C_{1-\alpha}([0, T])$. Due to Lemma 3.1 there exists a system of solutions $\left(u_{1}^{1}, u_{1}^{2}, \ldots, u_{1}^{n}\right) \in C([0, T])^{n}$ for system (3.15). Using induction we obtain the sequence $\left(u_{k}^{1}, u_{k}^{2}, \ldots, u_{k}^{n}\right) \in C([0, T])^{n}, k \in \mathbb{N}$, satisfying

$$
\left\{\begin{aligned}
& D^{\alpha} u_{k}^{1}(t)= f_{1}\left(t, u_{k-1}^{1}(t), u_{k-1}^{2}(t), \ldots, u_{k-1}^{n}(t)\right)+M_{1} u_{k-1}^{1}(t)+\sum_{i, j=2}^{n} M_{j} \delta_{j i} u_{k-1}^{i}(t) \\
&-M_{1} u_{k}^{1}(t)-\sum_{i, j=2}^{n} M_{j} \delta_{j i} u_{k}^{i}(t), \quad t \in(0, T] \\
& D^{\alpha} u_{k}^{s}(t)= f_{s}\left(t, u_{k-1}^{1}(t), u_{k-1}^{2}(t), \ldots, u_{k-1}^{n}(t)\right)+M_{1} u_{k-1}^{s}(t) \\
&+\left(\sum_{i=2}^{n} M_{i}-M_{s}\right) u_{k}^{s}(t)+M_{s}\left(u_{k-1}^{1}(t)+\sum_{i=2}^{n} u_{k}^{i}(t)-u_{k}^{s}(t)\right) \\
&-M_{1} u_{k}^{s}(t)-\left(\sum_{i=2}^{n} M_{i}-M_{s}\right) u_{k-1}^{s}(t) \\
&-M_{s}\left(u_{k}^{1}(t)+\sum_{i=2}^{n} u_{k-1}^{i}(t)-u_{k-1}^{s}(t)\right), \quad t \in(0, T], 2 \leq s \leq n, \\
&\left.t^{1-\alpha} u_{k}^{s}(t)\right|_{t=0}=x_{0}^{s}, \quad 1 \leq s \leq n .
\end{aligned}\right.
$$

Now, put $p_{1}^{1}=u_{1}^{1}-u_{0}^{1}, p_{1}^{s}=u_{0}^{s}-u_{1}^{s}, 2 \leq s \leq n$. From (3.12) and (3.15), for all $t \in(0, T]$, we obtain

$$
\begin{aligned}
D^{\alpha} p_{1}^{1}(t)= & D^{\alpha} u_{1}^{1}(t)-D^{\alpha} u_{0}^{1}(t) \\
= & f_{1}\left(t, u_{0}^{1}(t), u_{0}^{2}(t), \ldots, u_{0}^{n}(t)\right)+M_{1} u_{0}^{1}(t)+\sum_{i, j=2}^{n} M_{j} \delta_{j i} u_{0}^{i}(t) \\
& -M_{1} u_{1}^{1}(t)-\sum_{i, j=2}^{n} M_{j} \delta_{j i} u_{1}^{i}(t)-D^{\alpha} u_{0}^{1}(t) \\
\geq & -M_{1} p_{1}^{1}(t)+\sum_{i, j=2}^{n} M_{j} \delta_{j i} p_{1}^{i}(t), \\
D^{\alpha} p_{1}^{s}(t)= & D^{\alpha} u_{0}^{s}(t)-D^{\alpha} u_{1}^{s}(t) \\
= & D^{\alpha} u_{0}^{s}(t)-f_{s}\left(t, u_{0}^{1}(t), u_{0}^{2}(t), \ldots, u_{0}^{n}(t)\right)-M_{1} u_{0}^{s}(t) \\
& -\left(\sum_{i=2}^{n} M_{i}-M_{s}\right) u_{1}^{s}(t)-M_{s}\left(u_{0}^{1}(t)+\sum_{i=2}^{n} u_{1}^{i}(t)-u_{1}^{s}(t)\right)+M_{1} u_{1}^{s}(t) \\
& +\left(\sum_{i=2}^{n} M_{i}-M_{s}\right) u_{0}^{s}(t)+M_{s}\left(u_{1}^{1}(t)+\sum_{i=2}^{n} u_{0}^{i}(t)-u_{0}^{s}(t)\right) \\
\geq & -M_{1} p_{1}^{s}(t)+\left(\sum_{i=2}^{n} M_{i}-M_{s}\right) p_{1}^{s}(t)+M_{s}\left(\sum_{i=1}^{n} p_{1}^{i}(t)-p_{1}^{s}(t)\right) \quad \text { for all } 2 \leq s \leq n, \\
\left.t^{1-\alpha} p_{1}^{1}(t)\right|_{t=0}= & \left.t^{1-\alpha} u_{1}^{1}(t)\right|_{t=0}-\left.t^{1-\alpha} u_{0}^{1}(t)\right|_{t=0} \geq x_{0}^{1}-x_{0}^{1}=0, \\
\left.t^{1-\alpha} p_{1}^{s}(t)\right|_{t=0}= & \left.t^{1-\alpha} u_{0}^{s}(t)\right|_{t=0}-\left.t^{1-\alpha} u_{1}^{s}(t)\right|_{t=0} \geq x_{0}^{s}-x_{0}^{s}=0, \quad 2 \leq s \leq n .
\end{aligned}
$$


Hence, using Theorem 3.1, we have

$$
u_{1}^{s} \leq u_{0}^{s}, \quad 2 \leq s \leq n
$$

and

$$
u_{1}^{1}-u_{0}^{1}+\sum_{i=2}^{n}\left(u_{0}^{i}-u_{1}^{i}\right) \geq u_{0}^{s}-u_{1}^{s}, \quad 2 \leq s \leq n .
$$

Consider now $q_{1}=\sum_{i=2}^{n} u_{1}^{i}-u_{1}^{1}$. Using (3.14) and (3.15) we have

$$
\begin{aligned}
& D^{\alpha} q_{1}(t)=\sum_{s=2}^{n} u_{1}^{s}(t)-u_{1}^{1}(t)=\sum_{s=2}^{n} D^{\alpha} u_{1}^{s}(t)-D^{\alpha} u_{1}^{1}(t) \\
& =\sum_{s=2}^{n} f_{s}\left(t, u_{0}^{1}(t), u_{0}^{2}(t), \ldots, u_{0}^{n}(t)\right)+\sum_{s=2}^{n} M_{1} u_{0}^{s}(t) \\
& +\sum_{s=2}^{n}\left(\sum_{i=2}^{n} M_{i}-M_{s}\right) u_{1}^{s}(t)+\sum_{s=2}^{n} M_{s}\left(u_{0}^{1}(t)+\sum_{i=2}^{n} u_{1}^{i}(t)-u_{1}^{s}(t)\right) \\
& -\sum_{s=2}^{n} M_{1} u_{1}^{s}(t)-\sum_{s=2}^{n}\left(\sum_{i=2}^{n} M_{i}-M_{s}\right) u_{0}^{s}(t) \\
& -\sum_{s=2}^{n} M_{s}\left(u_{1}^{1}(t)+\sum_{i=2}^{n} u_{0}^{i}(t)-u_{0}^{s}(t)\right)-f_{1}\left(t, u_{0}^{1}(t), u_{0}^{2}(t), \ldots, u_{0}^{n}(t)\right) \\
& -M_{1} u_{0}^{1}(t)-\sum_{i, j=2}^{n} M_{j} \delta_{j i} u_{0}^{i}(t)+M_{1} u_{1}^{1}(t)+\sum_{i, j=2}^{n} M_{j} \delta_{j i} u_{1}^{i}(t) \\
& =\sum_{s=2}^{n} f_{s}\left(t, u_{0}^{1}(t), u_{0}^{2}(t), \ldots, u_{0}^{n}(t)\right)-f_{1}\left(t, u_{0}^{1}(t), u_{0}^{2}(t), \ldots, u_{0}^{n}(t)\right) \\
& -\left(M_{1}-\sum_{s=2}^{n} M_{s}\right) q_{1}(t)+\left(M_{1}-\sum_{s=2}^{n} M_{s}\right)\left(\sum_{s=2}^{n} u_{0}^{s}(t)-u_{0}^{1}(t)\right) \\
& \geq-\left(M_{1}-\sum_{s=2}^{n} M_{s}\right) q_{1}(t)
\end{aligned}
$$

Moreover, (1.2) implies

$$
\left.t^{1-\alpha} q_{1}(t)\right|_{t=0}=\left.\sum_{i=2}^{n} t^{1-\alpha} u_{1}^{i}(t)\right|_{t=0}-\left.t^{1-\alpha} u_{1}^{1}(t)\right|_{t=0}=\sum_{i=2}^{n} x_{0}^{i}-x_{0}^{1} \geq 0 .
$$

Now, from Lemma 2.2 we conclude

$$
u_{1}^{1}(t) \leq \sum_{i=2}^{n} u_{1}^{i}(t) \quad \text { for all } t \in[0, T]
$$

Combining (3.17) and (3.18) with (3.19) we obtain for all $2 \leq s \leq n$ the inequalities

$$
u_{0}^{1}-\left(\sum_{i=2}^{n} u_{0}^{i}-u_{0}^{s}\right) \leq u_{1}^{1}-\left(\sum_{i=2}^{n} u_{1}^{i}-u_{1}^{s}\right) \leq u_{1}^{s} \leq u_{0}^{s} .
$$


Let $2 \leq s \leq n$ be fixed and suppose now that for some $k \in \mathbb{N}$ the following inequalities hold:

$$
u_{k-1}^{1}-\left(\sum_{i=2}^{n} u_{k-1}^{i}-u_{k-1}^{s}\right) \leq u_{k}^{1}-\left(\sum_{i=2}^{n} u_{k}^{i}-u_{k}^{s}\right) \leq u_{k}^{s} \leq u_{k-1}^{s} .
$$

Denote $p_{k+1}^{1}=u_{k+1}^{1}-u_{k}^{1}, p_{k+1}^{s}=u_{k}^{s}-u_{k+1}^{s}, 2 \leq s \leq n$. From (3.13), (3.16), and (3.20) we obtain

$$
\begin{aligned}
& D^{\alpha} p_{k+1}^{1}(t)=D^{\alpha} u_{k+1}^{1}(t)-D^{\alpha} u_{k}^{1}(t) \\
& =f_{1}\left(t, u_{k}^{1}(t), u_{k}^{2}(t), \ldots, u_{k}^{n}(t)\right)+M_{1} u_{k}^{1}(t)+\sum_{i, j=2}^{n} M_{j} \delta_{j i} u_{k}^{i}(t)-M_{1} u_{k+1}^{1}(t) \\
& -\sum_{i, j=2}^{n} M_{j} \delta_{j i} u_{k+1}^{i}(t)-f_{1}\left(t, u_{k-1}^{1}(t), u_{k-1}^{2}(t), \ldots, u_{k-1}^{n}(t)\right)-M_{1} u_{k-1}^{1}(t) \\
& -\sum_{i, j=2}^{n} M_{j} \delta_{j i} u_{k-1}^{i}(t)+M_{1} u_{k}^{1}(t)+\sum_{i, j=2}^{n} M_{j} \delta_{j i} u_{k}^{i}(t) \\
& \geq-M_{1}\left(u_{k 1}^{1}(t)-u_{k-1}^{1}(t)\right)-\sum_{i, j=2}^{n} M_{j} \delta_{j i}\left(u_{k}^{i}(t)-u_{k-1}^{i}(t)\right)+M_{1} u_{k}^{1}(t) \\
& +\sum_{i, j=2}^{n} M_{j} \delta_{j i} u_{k}^{i}(t)-M_{1} u_{k+1}^{1}(t)-\sum_{i, j=2}^{n} M_{j} \delta_{j i} u_{k+1}^{i}(t)-M_{1} u_{k-1}^{1}(t) \\
& -\sum_{i, j=2}^{n} M_{j} \delta_{j i} u_{k-1}^{i}(t)+M_{1} u_{k}^{1}(t)+\sum_{i, j=2}^{n} M_{j} \delta_{j i} u_{k}^{i}(t) \\
& =-M_{1} p_{k+1}^{1}(t)+\sum_{i, j=2}^{n} M_{j} \delta_{j i} p_{k+1}^{i}(t), \\
& D^{\alpha} p_{k+1}^{s}(t)=D^{\alpha} u_{k}^{s}(t)-D^{\alpha} u_{k+1}^{s}(t) \\
& \geq\left(-M_{1}+\sum_{i=2}^{n} M_{i}-M_{s}\right)\left(u_{k-1}^{s}(t)-u_{k}^{s}(t)\right)-M_{s}\left(u_{k-1}^{1}(t)-u_{k}^{1}(t)\right. \\
& \left.+u_{k-1}^{s}(t)-u_{k}^{s}(t)-\sum_{i=2}^{n}\left(u_{k-1}^{i}-u_{k}^{i}\right)\right)+M_{1} u_{k-1}^{s}(t)+\left(\sum_{i=2}^{n} M_{i}-M_{s}\right) u_{k}^{s}(t) \\
& +M_{s}\left(u_{k-1}^{1}(t)+\sum_{i=2}^{n} u_{k}^{i}(t)-u_{k}^{s}(t)\right)-M_{1} u_{k}^{s}(t)-\left(\sum_{i=2}^{n} M_{i}-M_{s}\right) u_{k-1}^{s}(t) \\
& -M_{s}\left(u_{k}^{1}(t)+\sum_{i=2}^{n} u_{k-1}^{i}(t)-u_{k-1}^{s}(t)\right)-M_{1} u_{k}^{s}(t)-\left(\sum_{i=2}^{n} M_{i}-M_{s}\right) u_{k+1}^{s}(t) \\
& -M_{s}\left(u_{k}^{1}(t)+\sum_{i=2}^{n} u_{k+1}^{i}(t)-u_{k+1}^{s}(t)\right)+M_{1} u_{k+1}^{s}(t)+\left(\sum_{i=2}^{n} M_{i}-M_{s}\right) u_{k}^{s}(t) \\
& +M_{s}\left(u_{k+1}^{1}(t)+\sum_{i=2}^{n} u_{k}^{i}(t)-u_{k}^{s}(t)\right) \\
& =-M_{1} p_{k+1}^{s}(t)+\left(\sum_{i=2}^{n} M_{i}-M_{s}\right) p_{k+1}^{s}(t)+M_{s}\left(\sum_{i=1}^{n} p_{k+1}^{i}(t)-p_{k+1}^{s}(t)\right) .
\end{aligned}
$$


Also observe that $\left.t^{1-\alpha} p_{k+1}^{1}(t)\right|_{t=0}=\left.t^{1-\alpha} p_{k+1}^{s}(t)\right|_{t=0}=0$, which, together with the above, due to Theorem 3.1, gives

$$
\begin{aligned}
& u_{k+1}^{s} \leq u_{k}^{s}, \quad 2 \leq s \leq n, \\
& u_{k}^{s}-u_{k+1}^{s} \leq \sum_{i=2}^{n}\left(u_{k}^{i}-u_{k+1}^{i}\right)+u_{k+1}^{1}-u_{k}^{1} .
\end{aligned}
$$

Consider now $q_{k}=\sum_{i=2}^{n} u_{k}^{i}-u_{k}^{1}$. Using the same arguments as with $q_{1}$ we obtain

$$
D^{\alpha} q_{k}(t) \geq-\left(M_{1}-\sum_{s=2}^{n} M_{s}\right) q_{k}(t)
$$

and

$$
\left.t^{1-\alpha} q_{k}(t)\right|_{t=0} \geq 0
$$

which, due to Lemma 2.2, gives

$$
u_{k}^{1} \leq \sum_{s=2}^{n} u_{k}^{i}
$$

Summarizing, by (3.21)-(3.23) and induction, we obtain the following inequalities describing the sequences $\left(u_{k}^{s}\right)_{k \in \mathbb{N} \cup\{0\}}$ :

$$
\begin{aligned}
u_{0}^{1}-\left(\sum_{i=2}^{n} u_{0}^{i}-u_{0}^{s}\right) & \leq u_{1}^{1}-\left(\sum_{i=2}^{n} u_{1}^{i}-u_{1}^{s}\right) \\
& \leq \cdots \leq u_{k}^{1}-\left(\sum_{i=2}^{n} u_{k}^{i}-u_{k}^{s}\right) \\
& \leq u_{k}^{s} \leq \cdots \leq u_{1}^{s} \leq u_{0}^{s},
\end{aligned}
$$

where $2 \leq s \leq n$. The inequalities (3.24) imply

$$
\lim _{k \rightarrow \infty} u_{k}^{s}(t)=\bar{u}^{s}(t), \quad s=2, \ldots, n .
$$

Observe that

$$
u_{0}^{1}-\left(\sum_{i=2}^{n} u_{0}^{i}-u_{0}^{s}\right) \leq \bar{u}^{s} \leq u_{0}^{s}, \quad s=2, \ldots, n .
$$

In order to show that the sequence $\left(u_{k}^{1}\right)$ is convergent observe first that from (3.24) there exists a function $x^{*}$ such that

$$
\lim _{k \rightarrow \infty}\left(u_{k}^{1}(t)-\sum_{i=2}^{n-1} u_{k}^{i}(t)\right)=x^{*}(t)
$$


Hence, putting $\bar{u}^{1}=x^{*}+\sum_{s=2}^{n-1} \bar{u}^{s}$, we have

$$
\begin{aligned}
\lim _{k \rightarrow \infty}\left(u_{k}^{1}(t)-\bar{u}^{1}(t)\right) & =\lim _{k \rightarrow \infty}\left(u_{k}^{1}(t)-x^{*}(t)-\sum_{s=2}^{n-1} \bar{u}^{s}(t)+\sum_{s=2}^{n-1} u_{k}^{s}(t)-\sum_{s=2}^{n-1} u_{k}^{s}(t)\right) \\
& =\lim _{k \rightarrow \infty}\left(u_{k}^{1}(t)-\sum_{s=2}^{n-1} u_{k}^{s}(t)-x^{*}(t)+\sum_{s=2}^{n-1}\left(u_{k}^{s}(t)-\bar{u}^{s}(t)\right)\right) \\
& =\lim _{k \rightarrow \infty}\left(u_{k}^{1}(t)-\sum_{s=2}^{n-1} u_{k}^{s}(t)-x^{*}(t)\right)+\sum_{s=2}^{n-1} \lim _{k \rightarrow \infty}\left(u_{k}^{s}(t)-\bar{u}^{s}(t)\right)=0 .
\end{aligned}
$$

In order to show the uniform convergence of sequences $\left(u_{k}^{2}\right),\left(u_{k}^{3}\right), \ldots,\left(u_{k}^{n}\right)$, observe that from (3.24) and from the fact that $u_{k}^{s} \rightarrow \bar{u}^{s}, s=2,3, \ldots, n$, we have

$$
\bar{u}^{s} \leq u_{k}^{s} \leq \cdots \leq u_{1}^{s} \leq u_{0}^{s} \quad \text { for all } k \in \mathbb{N} .
$$

Then, the uniform convergence of sequences $\left(u_{k}^{s}\right), s=2,3, \ldots, n$, on a compact subset of $(0, T]$ is a straightforward consequence of Dini's theorem, which states that if a monotone sequence of continuous functions is convergent on a compact set, then it converges uniformly.

Showing a uniform convergence of $\left(u_{k}^{1}\right)$ requires some observations. Take any $2 \leq s \leq n$ and denote

$$
h_{k}=u_{k}^{1}-\left(\sum_{i=2}^{n} u_{k}^{i}-u_{k}^{s}\right), \quad k \in \mathbb{N} \cup\{0\} .
$$

From (3.24) and the convergence of $\left(u_{k}^{1}\right), \ldots,\left(u_{k}^{n}\right)$ we have

$$
h_{0} \leq h_{1} \leq \cdots \leq h_{k} \leq \bar{u}^{1}-\left(\sum_{i=2}^{n} \bar{u}^{i}-\bar{u}^{s}\right) \text {. }
$$

Applying again Dini's result we get the uniform convergence of $\left(h_{k}\right)$ on every compact subset of $(0, T]$. Finally note that

$$
u_{k}^{1}=h_{k}+\left(\sum_{i=2}^{n} u_{k}^{i}-u_{k}^{s}\right), \quad k \in \mathbb{N},
$$

and thus $\left(u_{k}^{1}\right)$ is uniformly convergent on a compact subset of $(0, T]$ to $\bar{u}^{1}$ as a linear combination of sequences uniformly convergent.

Moreover, observe that the limit functions satisfy the properties

$$
\begin{aligned}
& (n-1) u_{0}^{1}-(n-2) \sum_{i=2}^{n} u_{0}^{i} \leq \bar{u}^{1} \leq \sum_{i=2}^{n} u_{0}^{i}, \\
& u_{0}^{1}-\sum_{i=2}^{n} u_{0}^{i}+u_{0}^{s} \leq \bar{u}^{s} \leq u_{0}^{s}, \quad 2 \leq s \leq n .
\end{aligned}
$$


Taking $k$ to $\infty$ in (3.16) we see that $\left(\bar{u}^{1}, \bar{u}^{2}, \ldots, \bar{u}^{n}\right)$ is a system of solutions of system (1.1). Also observe that from (3.24) we have the following relations between the limit functions:

$$
\bar{u}^{1}-\left(\sum_{i=2}^{n} \bar{u}^{i}-\bar{u}^{s}\right) \leq \bar{u}^{s}, \quad 2 \leq s \leq n
$$

which ends the proof.

Remark 3.1 Observe that using the same methods as in the proof of Theorem 3.2 we can see that $\left(\bar{u}^{1}, \bar{u}^{2}, \ldots, \bar{u}^{n}\right)$ is an extremal solution of system $(1.1)$ in the sense that if $\left(u^{1}, \ldots, u^{n}\right)$ were any other solution such that

$$
u_{0}^{1}-\left(\sum_{i=2}^{n} u_{0}^{i}-u_{0}^{s}\right) \leq u^{1}-\left(\sum_{i=2}^{n} u^{i}-u^{s}\right) \leq u_{0}^{s}, \quad u_{0}^{1}-\left(\sum_{i=2}^{n} u_{0}^{i}-u_{0}^{s}\right) \leq u^{s} \leq u_{0}^{s}
$$

for any $2 \leq s \leq n$, then we would have

$$
\bar{u}^{1}-\left(\sum_{i=2}^{n} \bar{u}^{i}-\bar{u}^{s}\right) \leq u^{1}-\left(\sum_{i=2}^{n} u^{i}-u^{s}\right), \quad u^{s} \leq \bar{u}^{s}, 2 \leq s \leq n .
$$

\section{The system of three fractional differential equations}

In order to see the nature of the iterative procedure introduced in the proof of Theorem 3.2, we consider the case $n=3$.

Corollary 4.1 If there exist $u_{0}, v_{0}, w_{0} \in C_{1-\alpha}([0, T]), u_{0} \leq v_{0}+w_{0}$ such that

$$
\begin{cases}D^{\alpha} u_{0}(t) \leq f\left(t, u_{0}(t), v_{0}(t), w_{0}(t)\right), & t \in(0, T], \\ D^{\alpha} v_{0}(t) \geq g\left(t, u_{0}(t), v_{0}(t), w_{0}(t)\right), & t \in(0, T], \\ D^{\alpha} w_{0}(t) \geq h\left(t, u_{0}(t), v_{0}(t), w_{0}(t)\right), & t \in(0, T], \\ \left.t^{1-\alpha} u_{0}(t)\right|_{t=0} \leq x_{0}, & \\ \left.t^{1-\alpha} v_{0}(t)\right|_{t=0} \geq y_{0}, & \\ \left.t^{1-\alpha} w_{0}(t)\right|_{t=0} \geq z_{0}, & \end{cases}
$$

and there exist $M \in \mathbb{R}, N, S \geq 0$ satisfying

$$
\begin{aligned}
& f\left(t, \alpha_{1}, \alpha_{2}, \alpha_{3}\right)-f\left(t, \beta_{1}, \beta_{2}, \beta_{n}\right) \geq-M\left(\alpha_{1}-\beta_{1}\right)+(-N+S)\left(\alpha_{2}-\beta_{2}\right)+(N-S)\left(\alpha_{3}-\beta_{3}\right), \\
& g\left(t, \alpha_{1}, \alpha_{2}, \alpha_{3}\right)-g\left(t, \beta_{1}, \beta_{2}, \beta_{3}\right) \geq-N\left(\alpha_{1}-\beta_{1}\right)+(-M+S)\left(\alpha_{2}-\beta_{2}\right)+N\left(\alpha_{3}-\beta_{3}\right), \\
& h\left(t, \alpha_{1}, \alpha_{2}, \alpha_{3}\right)-h\left(t, \beta_{1}, \beta_{2}, \beta_{3}\right) \geq-S\left(\alpha_{1}-\beta_{1}\right)+S\left(\alpha_{2}-\beta_{2}\right)+(-M+N)\left(\alpha_{3}-\beta_{3}\right),
\end{aligned}
$$

where $\alpha_{i}, \beta_{i} \in \mathbb{R}, 1 \leq i \leq 3$ satisfy, for all $t \in[0, T]$,

$$
\begin{array}{ll}
u_{0}(t)-w_{0}(t) \leq \beta_{1}-\beta_{3} \leq \alpha_{1}-\alpha_{3} \leq v_{0}(t), & u_{0}(t)-w_{0}(t) \leq \alpha_{2} \leq \beta_{2} \leq v_{0}(t), \\
u_{0}(t)-v_{0}(t) \leq \beta_{1}-\beta_{2} \leq \alpha_{1}-\alpha_{2} \leq w_{0}(t), & u_{0}(t)-v_{0}(t) \leq \alpha_{3} \leq \beta_{3} \leq w_{0}(t)
\end{array}
$$

and

$$
(g+h-f)(t, u, v, w) \geq(-M+N+S)(v+w-u),
$$


where

$$
\begin{aligned}
& u_{0}(t)-w_{0}(t) \leq u-w \leq v \leq v_{0}(t) \\
& u_{0}(t)-v_{0}(t) \leq u-v \leq w \leq w_{0}(t)
\end{aligned}
$$

Then there exists a solution

$$
\left(u^{*}, v^{*}, w^{*}\right) \in\left[2 u_{0}-v_{0}-w_{0}, v_{0}+w_{0}\right] \times\left[u_{0}-w_{0}, v_{0}\right] \times\left[u_{0}-v_{0}, w_{0}\right]
$$

of (4.1) and the sequences $\left(u_{n}\right) \subseteq\left[2 u_{0}-v_{0}-w_{0}, v_{0}+w_{0}\right],\left(v_{n}\right) \subseteq\left[u_{0}-w_{0}, v_{0}\right],\left(w_{n}\right) \subseteq$ $\left[u_{0}-v_{0}, w_{0}\right]$ such that $u_{n} \rightarrow u^{*}, v_{n} \rightarrow v^{*}, w_{n} \rightarrow w^{*}$ uniformly on compact subsets of $(0, T]$. Moreover, the following inequalities hold:

$$
\begin{aligned}
& u_{0}-v_{0} \leq u_{1}-v_{1} \leq \cdots \leq u_{n}-v_{n} \leq \cdots \leq u^{*}-v^{*} \leq w^{*} \leq \cdots \leq w_{n} \leq \cdots \leq w_{1} \leq w_{0} \\
& u_{0}-w_{0} \leq u_{1}-w_{1} \leq \cdots \leq u_{n}-w_{n} \leq \cdots \leq u^{*}-w^{*} \leq v^{*} \leq \cdots \leq v_{n} \leq \cdots \leq v_{1} \leq v_{0}
\end{aligned}
$$

\subsection{Example}

Consider the nonlinear problem of the form

$$
\left\{\begin{array}{l}
D^{0.5} u(t)=\Gamma(1.5)^{-1} v(t)-\Gamma(1.5)^{-1} w(t)+(v(t)-t)^{2}+2(t-w(t)+u(t))^{2}, \\
D^{0.5} v(t)=\Gamma(1.5)^{-1} v(t)+(v(t)-t)^{2}+(t-w(t)+u(t))^{2}, \\
D^{0.5} w(t)=-\Gamma(1.5)^{-1} u(t)+\Gamma(1.5)^{-1} v(t)+(t-w(t)+u(t))^{2}, \\
\left.t^{0.5} u(t)\right|_{t=0}=\left.t^{0.5} v(t)\right|_{t=0}=\left.t^{0.5} w(t)\right|_{t=0}=0,
\end{array}\right.
$$

where $t \in[0,1]$. Taking

$$
\begin{aligned}
& f(t, u, v, w)=\Gamma(1.5)^{-1} v-\Gamma(1.5)^{-1} w+(v-t)^{2}+2(t-w+u)^{2}, \\
& g(t, u, v, w)=\Gamma(1.5)^{-1} v+(v-t)^{2}+(t-w+u)^{2}, \\
& h(t, u, v, w)=-\Gamma(1.5)^{-1} u+\Gamma(1.5)^{-1} v+(t-w+u)^{2}
\end{aligned}
$$

and

$$
u_{0}(t)=0, \quad v_{0}(t)=w_{0}(t)=t, \quad t \in[0,1]
$$

we obtain, for all $t \in[0,1]$,

$$
\begin{aligned}
& D^{0.5} u_{0}(t)=0=f\left(t, u_{0}(t), v_{0}(t), w_{0}(t)\right) \\
& D^{0.5} v_{0}(t)=\frac{\sqrt{t}}{\Gamma(1.5)} \geq \frac{t}{\Gamma(1.5)}=g\left(t, u_{0}(t), v_{0}(t), w_{0}(t)\right) \\
& D^{0.5} w_{0}(t)=\frac{\sqrt{t}}{\Gamma(1.5)} \geq 0=h\left(t, u_{0}(t), v_{0}(t), w_{0}(t)\right)
\end{aligned}
$$

Next, for all $\alpha_{i}, \beta_{i} \in \mathbb{R}, 1 \leq i \leq 3$ such that

$$
\begin{array}{ll}
-t \leq \beta_{1}-\beta_{3} \leq \alpha_{1}-\alpha_{3} \leq t, & -t \leq \alpha_{2} \leq \beta_{2} \leq t \\
-t \leq \beta_{1}-\beta_{2} \leq \alpha_{1}-\alpha_{2} \leq t, & -t \leq \alpha_{3} \leq \beta_{3} \leq t
\end{array}
$$


one can calculate that

$$
\begin{aligned}
& f\left(t, \alpha_{1}, \alpha_{2}, \alpha_{3}\right)-f\left(t, \beta_{1}, \beta_{2}, \beta_{3}\right) \geq \Gamma(1.5)^{-1}\left(\alpha_{2}-\beta_{2}\right)-\Gamma(1.5)^{-1}\left(\alpha_{3}-\beta_{3}\right), \\
& g\left(t, \alpha_{1}, \alpha_{2}, \alpha_{3}\right)-g\left(t, \beta_{1}, \beta_{2}, \beta_{3}\right) \geq \Gamma(1.5)^{-1}\left(\alpha_{2}-\beta_{2}\right), \\
& h\left(t, \alpha_{1}, \alpha_{2}, \alpha_{3}\right)-h\left(t, \beta_{1}, \beta_{2}, \beta_{3}\right) \geq-\Gamma(1.5)^{-1}\left(\alpha_{1}-\beta_{1}\right)+\Gamma(1.5)^{-1}\left(\alpha_{2}-\beta_{2}\right) .
\end{aligned}
$$

Therefore it is sufficient to take in Corollary $4.1 M=0, N=0, S=\Gamma(1.5)^{-1}$. Finally observe that condition (4.2) also holds. Thus, the system of fractional differential equations (4.3) has a solution $\left(u^{*}, v^{*}, w^{*}\right) \in[-2 t, 2 t] \times[-t, t] \times[-t, t]$.

Now, using the proof of Theorem 3.2 and Lemma 3.1, we can derive the iterative procedure $\left(u_{k}, v_{k}, w_{k}\right)$ convergent to the solution $\left(u^{*}, v^{*}, w^{*}\right)$. First observe that the sequences $\left(u_{k}\right),\left(v_{k}\right),\left(w_{k}\right)$ satisfy the following system of linear equations:

$$
\begin{aligned}
& D^{0.5} u_{k}=f\left(t, u_{k-1}, v_{k-1}, w_{k-1}\right)-\Gamma(1.5)^{-1} v_{k-1}+\Gamma(1.5)^{-1} w_{k-1}+\Gamma(1.5)^{-1} v_{k}-\Gamma(1.5)^{-1} w_{k}, \\
& D^{0.5} v_{k}=g\left(t, u_{k-1}, v_{k-1}, w_{k-1}\right)-\Gamma(1.5)^{-1} v_{k-1}+\Gamma(1.5)^{-1} v_{k}, \\
& D^{0.5} w_{k}=h\left(t, u_{k-1}, v_{k-1}, w_{k-1}\right)+\Gamma(1.5)^{-1} u_{k-1}-\Gamma(1.5)^{-1} v_{k-1}-\Gamma(1.5)^{-1} u_{k}+\Gamma(1.5)^{-1} v_{k}, \\
& \left.t^{0.5} u_{k}(t)\right|_{t=0}=\left.t^{0.5} v_{k}(t)\right|_{t=0}=\left.t^{0.5} w_{k}(t)\right|_{t=0}=0
\end{aligned}
$$

which can be equivalently transformed to the system

$$
\left\{\begin{array}{l}
u_{k}+v_{k}+w_{k}=p_{k}, \\
u_{k}-v_{k}+w_{k}=q_{k}, \\
u_{k}+v_{k}-w_{k}=r_{k},
\end{array}\right.
$$

where $p_{k}, q_{k}, r_{k}$ are the solutions of the following systems:

$$
\begin{aligned}
& \left\{\begin{aligned}
& D^{0.5} p_{k}=(f+g+h)\left(t, u_{k-1}, v_{k-1}, w_{k-1}\right) \\
&+\Gamma(1.5)^{-1} u_{k-1}-3 \Gamma(1.5)^{-1} v_{k-1}+\Gamma(1.5)^{-1} w_{k-1}-\Gamma(1.5)^{-1} p_{k}, \\
&\left.t^{0.5} p_{k}(t)\right|_{t=0}=0
\end{aligned}\right. \\
& \left\{\begin{aligned}
& D^{0.5} q_{k}=(f-g+h)\left(t, u_{k-1}, v_{k-1}, w_{k-1}\right) \\
&+\Gamma(1.5)^{-1} u_{k-1}-\Gamma(1.5)^{-1} v_{k-1}+\Gamma(1.5)^{-1} w_{k-1}-\Gamma(1.5)^{-1} q_{k}, \\
&\left.t^{0.5} q_{k}(t)\right|_{t=0}=0
\end{aligned}\right. \\
& \left\{\begin{aligned}
& D^{0.5} r_{k}=(f+g-h)\left(t, u_{k-1}, v_{k-1}, w_{k-1}\right) \\
& \quad-\Gamma(1.5)^{-1} u_{k-1}-\Gamma(1.5)^{-1} v_{k-1}+\Gamma(1.5)^{-1} w_{k-1}+\Gamma(1.5)^{-1} r_{k}, \\
&\left.t^{0.5} r_{k}(t)\right|_{t=0}=0 .
\end{aligned}\right.
\end{aligned}
$$

The solutions of the above systems, due to Lemma 2.1, are given by the formulas

$$
\begin{aligned}
p_{k}(t)= & \int_{0}^{t}(t-s)^{-0.5} E_{0.5,0.5}\left(-\Gamma(1.5)^{-1}(t-s)^{0.5}\right)\left((f+g+h)\left(s, u_{k-1}(s), v_{k-1}(s), w_{k-1}(s)\right)\right. \\
& \left.+\Gamma(1.5)^{-1} u_{k-1}(s)-3 \Gamma(1.5)^{-1} v_{k-1}(s)+\Gamma(1.5)^{-1} w_{k-1}(s)\right) d s, \\
q_{k}(t)= & \int_{0}^{t}(t-s)^{-0.5} E_{0.5,0.5}\left(-\Gamma(1.5)^{-1}(t-s)^{0.5}\right)\left((f-g+h)\left(s, u_{k-1}(s), v_{k-1}(s), w_{k-1}(s)\right)\right. \\
& \left.+\Gamma(1.5)^{-1} u_{k-1}(s)-\Gamma(1.5)^{-1} v_{k-1}(s)+\Gamma(1.5)^{-1} w_{k-1}(s)\right) d s,
\end{aligned}
$$




$$
\begin{aligned}
r_{k}(t)= & \int_{0}^{t}(t-s)^{-0.5} E_{0.5,0.5}\left(\Gamma(1.5)^{-1}(t-s)^{0.5}\right)\left((f+g-h)\left(s, u_{k-1}(s), v_{k-1}(s), w_{k-1}(s)\right)\right. \\
& \left.-\Gamma(1.5)^{-1} u_{k-1}(s)-\Gamma(1.5)^{-1} v_{k-1}(s)+\Gamma(1.5)^{-1} w_{k-1}(s)\right) d s .
\end{aligned}
$$

In consequence, the iterative sequences are of the form

$$
\begin{aligned}
u_{k}(t)= & \frac{1}{2}\left(q_{k}+r_{k}\right) \\
= & \frac{1}{2} \int_{0}^{t}(t-s)^{-0.5}\left[E _ { 0 . 5 , 0 . 5 } ( - \Gamma ( 1 . 5 ) ^ { - 1 } ( t - s ) ^ { 0 . 5 } ) \left((f-g+h)\left(s, u_{k-1}(s), v_{k-1}(s), w_{k-1}(s)\right)\right.\right. \\
& \left.+\Gamma(1.5)^{-1} u_{k-1}(s)-\Gamma(1.5)^{-1} v_{k-1}(s)+\Gamma(1.5)^{-1} w_{k-1}(s)\right) \\
& +E_{\alpha, \alpha}\left(\Gamma 1.5^{-1}(t-s)^{\alpha}\right)\left((f+g-h)\left(s, u_{k-1}(s), v_{k-1}(s), w_{k-1}(s)\right)\right. \\
& \left.\left.-\Gamma(1.5)^{-1} u_{k-1}(s)-\Gamma(1.5)^{-1} v_{k-1}(s)+\Gamma(1.5)^{-1} w_{k-1}(s)\right)\right] d s, \\
v_{k}(t)= & \frac{1}{2}\left(p_{k}-q_{k}\right) \\
= & \frac{1}{2} \int_{0}^{t}(t-s)^{-0.5}\left[E _ { 0 . 5 , 0 . 5 } ( - \Gamma ( 1 . 5 ) ^ { - 1 } ( t - s ) ^ { 0 . 5 } ) \left((f+g+h)\left(s, u_{k-1}(s), v_{k-1}(s), w_{k-1}(s)\right)\right.\right. \\
& \left.+\Gamma(1.5)^{-1} u_{k-1}(s)-3 \Gamma(1.5)^{-1} v_{k-1}(s)+\Gamma(1.5)^{-1} w_{k-1}(s)\right) \\
& -E_{0.5,0.5}\left(-\Gamma(1.5)^{-1}(t-s)^{0.5}\right)\left((f-g+h)\left(s, u_{k-1}(s), v_{k-1}(s), w_{k-1}(s)\right)\right. \\
& \left.\left.+\Gamma(1.5)^{-1} u_{k-1}(s)-\Gamma(1.5)^{-1} v_{k-1}(s)+\Gamma(1.5)^{-1} w_{k-1}(s)\right)\right] d s, \\
w_{k}(t)= & \frac{1}{2}\left(p_{k}-r_{k}\right) \\
= & \frac{1}{2} \int_{0}^{t}(t-s)^{-0.5}\left[E _ { 0 . 5 , 0 . 5 } ( - \Gamma ( 1 . 5 ) ^ { - 1 } ( t - s ) ^ { 0 . 5 } ) \left((f+g+h)\left(s, u_{k-1}(s), v_{k-1}(s), w_{k-1}(s)\right)\right.\right. \\
& \left.+\Gamma(1.5)^{-1} u_{k-1}(s)-3 \Gamma(1.5)^{-1} v_{k-1}(s)+\Gamma(1.5)^{-1} w_{k-1}(s)\right) \\
& -E_{0.5,0.5}\left(\Gamma(1.5)^{-1}(t-s)^{0.5}\right)\left((f+g-h)\left(s, u_{k-1}(s), v_{k-1}(s), w_{k-1}(s)\right)\right. \\
& \left.\left.-\Gamma(1.5)^{-1} u_{k-1}(s)-\Gamma(1.5)^{-1} v_{k-1}(s)+\Gamma(1.5)^{-1} w_{k-1}(s)\right)\right] d s .
\end{aligned}
$$

\section{Competing interests}

The author declares that he has no competing interests.

\section{Author's contributions}

The author formulated and proved all the results in the article, produced the illustrative example, wrote the manuscript, and read and approved it.

\section{Acknowledgements}

The author is very grateful to the reviewers for the remarks, which improved the final version of the manuscript. This article was financially supported by University of Łódź as a part of donation for the research activities aimed at the development of young scientists, grant no. 545/1117.

Received: 7 January 2015 Accepted: 17 May 2015 Published online: 03 June 2015

\section{References}

1. Babakhani, A, Daftardar-Gejji, V: Existence of positive solutions of nonlinear fractional differential equations. J. Math. Anal. Appl. 278, 434-442 (2003)

2. Bai, ZB: On positive solutions of a nonlocal fractional boundary value problem. Nonlinear Anal. 72, 916-924 (2010)

3. Bai, ZB, Lu, HS: Positive solutions of boundary value problems of nonlinear fractional differential equation. J. Math Anal. Appl. 311, 495-505 (2005)

4. Belmekki, M, Benchohra, M: Existence results for fractional order semilinear functional differential equations with nondense domain. Nonlinear Anal. 72, 925-932 (2010) 
5. Belmekki, M, Nieto, JJ, Rodriguez-Lopez, R: Existence of periodic solution for a nonlinear fractional differential equation. Bound. Value Probl. 2009, Article ID 324561 (2009)

6. El-Shahed, M: Positive solutions for boundary value problem of nonlinear fractional differential equation. Abstr. Appl. Anal. 2007, Article ID 10368 (2007)

7. Lakshmikantham, V, Vatsala, AS: Basic theory of fractional differential equations. Nonlinear Anal. 69(8), 2677-2682 (2008)

8. Lakshmikanthan, V, Vatsala, AS: General uniqueness and monotone iterative technique for fractional differential equations. Appl. Math. Lett. 21, 828-834 (2008)

9. McRae, FA: Monotone iterative technique and existence results for fractional differential equations. Nonlinear Anal. 71, 6093-6096 (2009)

10. Wang, G, Agarwal, RP, Cabada, A: Existence results and the monotone iterative technique for systems of nonlinear fractional differential equations. Appl. Math. Lett. 25, 1019-1024 (2012)

11. Wei, Z, Li, Q, Che, J: Initial value problems for fractional differential equations involving Riemann-Liouville sequential fractional derivative. J. Math. Anal. Appl. 367, 260-272 (2010)

12. Zhang, S: Monotone iterative method for initial value problem involving Riemann-Liouville fractional derivatives. Nonlinear Anal. 71, 2087-2093 (2009)

13. Zhang, S: The existence of a positive solution for a nonlinear fractional differential equation. J. Math. Anal. Appl. 252, 804-812 (2000)

14. Ahmad, B, Nieto, JJ: Existence results for a coupled system of nonlinear fractional differential equations with three-point boundary conditions. Comput. Math. Appl. 58, 1838-1843 (2009)

15. Bai, C, Fang, J: The existence of a positive solution for a singular coupled system of nonlinear fractional differential equations. Appl. Math. Comput. 150, 611-621 (2004)

16. Bhalekar, S, Daftardar-Gejji, V: Fractional ordered Liu system with time-delay. Commun. Nonlinear Sci. Numer. Simul. $15,2178-2191(2010)$

17. Chen, Y, An, H: Numerical solutions of coupled Burgers equations with time and space fractional derivatives. Appl. Math. Comput. 200, 87-95 (2008)

18. Daftardar-Gejji, V: Positive solutions of a system of non-autonomous fractional differential equations. J. Math. Anal. Appl. 302, 56-64 (2005)

19. Gafiychuk, V, Datsko, B, Meleshko, V: Mathematical modeling of time fractional reaction-diffusion systems. J. Comput. Appl. Math. 220, 215-225 (2008)

20. Jafari, $H$, Daftardar-Gejii, V: Solving a system of nonlinear fractional differential equations using Adomian decomposition. J. Comput. Appl. Math. 196, 644-651 (2006)

21. Lazarevi, MP: Finite time stability analysis of $\mathrm{PD}^{\alpha}$ fractional control of robotic time-delay systems. Mech. Res. Commun. 33, 269-279 (2006)

22. Su, X: Boundary value problem for a coupled system of nonlinear fractional differential equations. Appl. Math. Lett. 22, 64-69 (2009)

23. Kilbas, AA, Srivastava, HM, Trujillo, JJ: Theory and Applications of Fractional Differential Equations. North-Holland Mathematics Studies, vol. 204. Elsevier, Amsterdam (2006)

24. Podlubny, I: Fractional Differential Equations. Mathematics in Science and Engineering. Academic Press, New York (1999)

25. Samko, SG, Kilbas, AA, Marichev, Ol: Fractional Integrals and Derivatives: Theory and Applications. Gordon \& Breach, Yverdon (1993)

\section{Submit your manuscript to a SpringerOpen ${ }^{\odot}$ journal and benefit from:}

$\checkmark$ Convenient online submission

- Rigorous peer review

Immediate publication on acceptance

Open access: articles freely available online

- High visibility within the field

- Retaining the copyright to your article 